\title{
FORESTIMATOR : UN PLUGIN QGIS D'ESTIMATION DE LA HAUTEUR DOMINANTE ET DU SITE INDEX DE PEUPLEMENTS RESINEUX A PARTIR DE LIDAR AERIEN
}

\author{
Laurent Dedry ${ }^{1}$, Olivier De Thier ${ }^{1}$, Jérôme Perin ${ }^{1}$, Adrien Michez ${ }^{1}$, Stéphanie Bonnet ${ }^{1}$, Philippe Lejeune ${ }^{1}$ \\ 1 : Université de Liège - Gembloux Agro-Bio Tech, Gembloux, p.lejeune@ulg.ac.be
}

\begin{abstract}
Résumé
La hauteur dominante est une information souvent utilisée en gestion forestière. II s'agit d'un bon indicateur du niveau de développement des peuplements, pouvant être employé pour estimer le niveau de productivité des peuplements purs équiennes. Cependant, son estimation à partir de mesures de terrain est relativement fastidieuse et coûteuse. Depuis plusieurs années, le LiDAR est reconnu pour sa capacité à estimer la hauteur des couverts forestiers de manière précise. En Wallonie, une couverture LiDAR basse densité $\left(0,8\right.$ points $/ \mathrm{m}^{2}$ au sol) est disponible sur l'intégralité du territoire. Cet article présente un outil d'aide à la gestion forestière s'appuyant sur un modèle de prédiction de la hauteur dominante à partir de données LiDAR aérien. Les estimations fournies par ce modèle sont ensuite couplées à des modèles de croissance en hauteur dominante qui permettent une mise à jour de la hauteur dominante au cours du temps et l'estimation du Site Index (indice de productivité). Cet outil a été validé pour les peuplements d'épicéa commun (Picea abies (L.) H. Karst.f) et de douglas (Pseudotsuga menziesii (Mirb.) Franco) sur une emprise géographique correspondant à la Wallonie (Belgique).Pour permettre une utilisation aisée, il a été intégré dans un plugin du logiciel de Système d'Information Géographique (SIG) open source QGIS. Gratuit et facile d'utilisation, il est destiné aux gestionnaires forestiers et aux scientifiques.
\end{abstract}

Mots-clés : LiDAR aérien, hauteur dominante, indice de productivité, SIG open source, QGIS, Wallonie

\begin{abstract}
In forestry, top height is a common parameter used as indicator of the stand development stage. It can be used to estimate the potential production of monospecific even-aged stands. However, accurate field estimation of top height is time-consuming and expensive. Since the last two decades, LiDAR has proven to be very useful in estimating forest heights. In Wallonia, a low density LiDAR dataset $\left(0.8\right.$ points $/ \mathrm{m}^{2}$ on ground-level) is available for the whole territory. This paper outlines a tool, based on a predictive model of top height from airborne LiDAR data, to help forest management decision-making. The estimations provided by the model are associated with top height growth models to update top height over time and then estimate Site Index. The model has been validated for Norway spruce (Picea abies (L.) H. Karst.) and Douglas-fir (Pseudotsuga menziesii (Mirb.) Franco) stands in the entire Wallonia area (Belgium). In order to facilitate access to these models, the process has been implemented as a plugin of the open source GIS software QGIS. Free and user-friendly, it is aimed to be used by forest managers and scientists.
\end{abstract}

Keywords: Airborne LiDAR, top height, Site Index, open source GIS, QGIS, Wallonia

\section{Introduction}

L'évaluation précise et continue de la ressource ligneuse est un préalable à la mise en place d'une gestion forestière durable. Classiquement, la caractérisation de cette ressource s'appuie sur des inventaires au sol réalisés par échantillonnage. Les arbres situés au sein d'un nombre limité de placettes font l'objet d'une série de mesures dendrométriques (diamètre à hauteur de poitrine, hauteur totale, hauteur de cime, etc.). Les mesures ainsi récoltées permettent, après l'application de méthodes de calcul appropriées, de produire des indicateurs décrivant la structure du peuplement, le stock de bois sur pied ou encore l'état de la régénération.

Certains indicateurs, indispensables à la mise au point de scénarios de gestion sylvicole, reposent généralement sur des mesures répétées dans le temps. C'est par exemple le cas de l'accroissement périodique en volume. II existe néanmoins des solutions plus simples pour estimer le potentiel de production d'un peuplement équienne dès lors que l'on connaît son âge et sa hauteur dominante (Hägglund, 1981 ; Sharma et al., 2002). La hauteur dominante (Hdom), définie comme la hauteur moyenne des 100 plus gros arbres par hectare (Rondeux, 1999), présente un intérêt tout particulier pour les gestionnaires forestiers car son évolution au cours du temps est, d'une part, fortement corrélée avec le potentiel de production des peuplements purs équiennes et, d'autre part, peu influencée par les opérations sylvicoles. La hauteur dominante, considérée à un âge de référence donné (50 ans dans le cas de peuplements résineux en Belgique), appelée aussi Site Index (ou indice de productivité), peut ainsi être utilisée pour estimer la productivité des peuplements forestiers sans avoir recours à de fastidieuses et coûteuses mesures dendrométriques répétées dans le temps sur des placettes permanentes. 
Depuis ses premières applications en foresterie dans le courant des années 90 (Nilsson, 1996 ; Naesset, 1997), le LiDAR (Light Detection And Ranging) a largement été étudié pour sa capacité à caractériser la ressource forestière et plus spécifiquement à mesurer des hauteurs, que ce soit à l'échelle du peuplement (Naesset et al, 2001), de la placette (Holmgren et al., 2003) ou de l'arbre (Persson et al., 2002 ; Popescu et al., 2004 ; Kaartinen et al., 2012). Le succès des applications LiDAR dans le domaine forestier s'explique en grande partie par sa capacité à fournir une vision tridimensionnelle du couvert forestier, alors que les autres techniques de télédétection se limitent généralement à fournir une représentation du toit de la canopée (Coops et al., 2004).

La précision des estimations de hauteurs dominantes dérivées du LiDAR est admise comme étant comparable à celle obtenue avec des mesures d'inventaires classiques (Naesset, 2004 ; Maltamo et al., 2009). Elle varie en fonction des paramètres d'acquisition (densité de points, altitude de vol, angle et fréquence d'émission), de la structure des peuplements, de la saison à laquelle s'effectue l'acquisition, des erreurs de positionnement sur le terrain, etc. (Naesset et al., 2014). Le LiDAR est maintenant utilisé de manière opérationnelle dans des inventaires forestiers de nombreux pays (White et al., 2013a ; Naesset 2007 ; Maltamo et al. 2011 ; Woods et al. 2011).

En raison de son coût relativement élevé, la répétition à intervalle régulier de vols LiDAR sur de larges territoires reste difficilement envisageable. Dans ce contexte, le développement d'outils permettant la mise à jour de données dérivées du LiDAR peut s'avérer particulièrement intéressante (White et al., 2013b). Par exemple, Watt et al. (2013) proposent des modèles permettant d'actualiser les estimations de hauteurs dominantes en combinant des données LiDAR avec des données satellites. Ces dernières, collectées annuellement, sont utilisées pour actualiser l'estimation de la hauteur dominante au cours du temps.

En Belgique, des modèles de croissance en hauteur dominante ont récemment été établis pour les peuplements purs équiennes d'épicéa, de douglas et de mélèzes (Perin et al., 2013 ; 2014). Ils permettent de prédire la croissance en hauteur dominante et le Site Index d'un peuplement lorsque la date de plantation et la hauteur dominante à un âge donné sont connues. Couplés à des estimations de hauteur dominante découlant de données LiDAR, ces modèles rendent possible une actualisation de ces dernières au cours du temps et l'évaluation du Site Index.

L'utilisation de logiciels open source tend à se développer fortement dans la communauté scientifique de même que dans le monde forestier (Morgenroth et al., 2013 ; Bastin et al., 2012). Les modalités d'accès aux données ont aussi fortement évolué, avec le développement des services Web. Parmi ces services on distingue notamment les Web Map Services (WMS) qui renvoient au client (l'utilisateur) une carte géoréférencée, produite sous forme d'image, au départ d'un serveur de données distant. Les Web Processing Service (WPS) quant à eux permettent à un client de soumettre l'exécution d'un géotraitement à un serveur distant. Ces nouveaux outils facilitent grandement le transfert standardisé d'informations et facilitent l'accès des utilisateurs aux résultats produits par les chercheurs.

Cet article présente un outil d'aide à la gestion forestière s'appuyant sur un modèle de prédiction de la hauteur dominante à partir de données LiDAR aérien. Les estimations de hauteurs dominantes découlant des données LiDAR sont ensuite couplées à des modèles de croissance en hauteur dominante préexistants qui permettent une mise à jour de la hauteur dominante au cours du temps et l'estimation du Site Index. Ces fonctionnalités ont été développées sous la forme d'une architecture client-serveur. Une extension (plugin) du logiciel SIG open source QGIS joue le rôle de client et un serveur associé à un système de gestion de base de données spatiale (PostGIS) réceptionne et traite les requêtes. L'outil a été développé pour l'épicéa commun (Picea abies (L.) H. Karst.) et le douglas (Pseudotsuga menziesii (Mirb.) Franco) pour une emprise géographique correspondant à la Wallonie (région au sud de la Belgique).

\section{Matériel}

\subsection{Zone d'étude}

La zone d'étude concerne l'ensemble du territoire de la Wallonie (Belgique). Elle couvre une surface de $16844 \mathrm{~km}^{2}$ (Figure 1). L'altitude y varie de $20 \mathrm{~m}$ à pratiquement $700 \mathrm{~m}$.

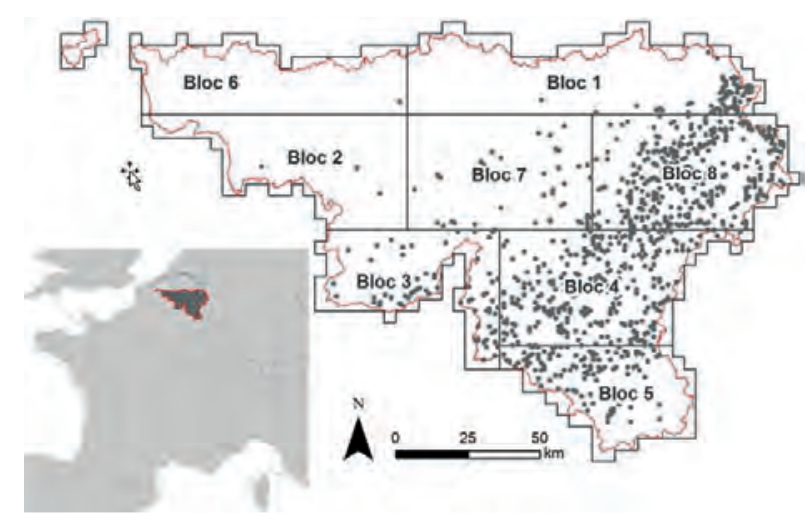

Figure 1: Plan de découpage des campagnes d'acquisition LiDAR en huit blocs. Distribution des placettes de l'Inventaire Permanent des Ressources Forestières de Wallonie utilisées pour la construction des modèles de hauteur dominante (points noirs).

La surface des forêts considérées comme productives est de $4753 \mathrm{~km}^{2}$ (28\% du territoire) répartie en $57 \%$ de peuplements feuillus, $40 \%$ de peuplements résineux et $3 \%$ de surface en attente de reboisement. L'épicéa commun et le douglas représentent respectivement $66 \%$ et $10 \%$ des peuplements résineux, auxquels il convient d'ajouter $6 \%$ de peuplements où ces 2 essences sont en mélange (Office Économique Wallon du Bois, 2013).

La sylviculture appliquée à ces 2 essences est majoritairement basée sur des plantations denses comportant entre 2000 et 3000 plants par ha. Ces peuplements équiennes sont régulièrement éclaircis 
dès l'âge de 20-25 ans et récoltés lorsqu'ils atteignent les dimensions d'exploitabilité (circonférences à hauteur de poitrine allant de 120 à $150 \mathrm{~cm}$ pour l'épicéa et supérieures à $180 \mathrm{~cm}$ pour le douglas). Les peuplements non éclaircis sont très rares en Wallonie.

\subsection{Données LiDAR}

En vue d'obtenir une couverture LiDAR complète de la Wallonie, huit blocs d'acquisition de données LiDAR ont été nécessaires (Figure 1). Les blocs 2, 3 et 6 ont été couverts au début de l'année 2013 tandis que les 5 autres blocs ont été acquis au début de l'année 2014. Les vols ont été réalisés de manière à obtenir une moyenne de 0,8 points $/ \mathrm{m}^{2}$ au sol. Les paramètres des acquisitions sont synthétisés dans le Tableau 1.

Les produits livrés par le prestataire correspondaient à un modèle numérique de terrain (MNT) et un modèle numérique de surface (MNS), tous deux réalisés à une résolution de $1 \mathrm{~m}$. Ces deux couches ont été soustraites l'une de l'autre (MNS - MNT) pour produire un modèle numérique de canopée (MNC) correspondant à la hauteur des éléments par rapport à la surface du sol.

\begin{tabular}{ll}
\hline Paramètre & Valeur \\
\hline Scanner & Riegl Litemapper 6800i \\
Altitude de vol & $1500 \mathrm{~m}$ \\
Vitesse & $75 \mathrm{~m} \cdot \mathrm{s}^{-1}$ \\
Recouvrement latéral & $30 \%$ \\
Fréquence d'émission & $150 \mathrm{kHz}$ \\
Angle de scan & $60^{\circ}$ \\
Nombre d'échos & 4 \\
Précision en $\mathrm{Z}$ & $12 \mathrm{~cm}$ \\
Précision en XY & $<1 \mathrm{~m}$ \\
\hline
\end{tabular}

Tableau 1 : Caractéristiques des acquisitions LiDAR.

\subsection{Données de hauteur dominante terrain}

Les données utilisées pour la construction des modèles d'estimation de la hauteur dominante à partir de données LiDAR sont issues de la base de données de I'Inventaire Permanent des Ressources Forestières de Wallonie (IPRFW). Cet inventaire régional repose sur un plan d'échantillonnage systématique non stratifié : les unités d'échantillonnage, de forme circulaire avec un rayon maximum de $18 \mathrm{~m}$, sont centrées sur les points d'une grille de $500 \mathrm{~m}$ (nord-sud) sur $1000 \mathrm{~m}$ (estouest) qui sont situés en forêt. Environ $10 \%$ des points sont remesurés chaque année en suivant un schéma garantissant une couverture uniforme du territoire (Rondeux et al., 2010). La hauteur dominante calculée sur ces placettes correspond à la hauteur moyenne arithmétique des 3 arbres dont la circonférence est la plus proche de la circonférence dominante, elle-même calculée, sur base de la moyenne arithmétique des 100 plus grosses tiges par hectare au sein de la placette.

Les placettes mesurées entre 2008 et 2014 dans lesquelles l'épicéa ou le douglas représentaient plus de $80 \%$ de la surface terrière ont été extraites de la base de données de l'inventaire. Les placettes ne comportant pas d'information sur la hauteur dominante ou l'âge de plantation ont été supprimées du jeu de données. Une phase de photo-interprétation a également permis d'identifier et d'éliminer les placettes mises à blanc entre le passage sur le terrain et le vol LiDAR ainsi que les placettes dont la localisation était en discordance avec les informations relevées sur le terrain. Au total, 674 placettes en épicéa et 97 placettes en douglas ont été sélectionnées. Ces placettes sont représentatives des classes d'âges et des conditions de croissance de ces deux essences dans la zone d'étude (Tableau 2).

Afin d'actualiser les mesures de hauteur dominante à la date d'acquisition des données LiDAR, les modèles de croissance développés par Perin et al. $(2013$; 2014) pour les peuplements équiennes d'épicéa et de douglas (équation 4) ont été utilisés. L'année à laquelle s'effectue l'actualisation de la hauteur dominante est déterminée par la position de la placette par rapport aux blocs d'acquisition LiDAR: les placettes situées dans les blocs 2, 3 et 6 sont actualisées à l'année 2013 tandis que les placettes situées dans les autres blocs sont actualisées à l'année 2014.

La répartition des jeux de données pour la calibration et la validation des modèles s'est effectuée en classant les placettes en fonction de leur date de mesure sur le terrain pour chaque essence. Ensuite, une placette sur trois a été sélectionnée pour le jeu de validation et deux sur trois pour le jeu de calibration de sorte que les deux jeux de données soient représentatifs des différentes années de mesure. Au total, le jeu de calibration est constitué de 449 placettes pour l'épicéa et de 65 placettes pour le douglas. Quant au jeu de validation, il comprend 225 placettes en épicéa et 32 en douglas.

\begin{tabular}{lcccc}
\hline Caractéristique & Moyenne & Ecart-type & Min & Max \\
\hline Altitude $(m)$ & 424 & 106 & 96 & 677 \\
$\begin{array}{l}\text { Pente }\left(^{\circ}\right) \\
\begin{array}{l}\text { Âge depuis la } \\
\text { plantation } \\
\text { (années) }\end{array}\end{array}$ & 6,9 & 6,3 & 0,0 & 37,0 \\
$\begin{array}{l}\text { Hauteur } \\
\text { dominante }(m)\end{array}$ & 23,8 & 19 & 10 & 117 \\
\hline
\end{tabular}

Tableau 2 : Caractéristiques des placettes d'épicéa et de douglas extraites de la base de données de I'IPRFW.

\section{Méthode}

\subsection{Estimation de la hauteur dominante à partir de données LiDAR}

Pour chaque placette extraite de la base de données de I'IPRFW ainsi que pour l'ensemble du territoire wallon, les arbres dominants ont été détectés à l'aide de la fonction CanopyMaxima du logiciel FUSION (McGaughey, 2013) sur le MNC. Cet algorithme s'appuie sur l'hypothèse que les maxima locaux d'un MNC correspondent généralement aux apex des arbres (Popescu et al., 2002 ; 2004). La fonction CanopyMaxima a été utilisée avec une fenêtre fixe de $5 \mathrm{~m}$ et en considérant un seuil de $4 \mathrm{~m}$ de hauteur en dessous duquel aucun maxima n'est détecté.

Une « hauteur dominante LiDAR » (Hdom LiDAR) a été calculée à l'échelle des différentes placettes. Elle 
correspond à la moyenne arithmétique des 100 plus hauts maxima locaux par hectare. Néanmoins, les faisceaux LiDAR interceptent rarement les bourgeons apicaux des arbres en raison de leur petite taille de sorte que la hauteur mesurée avec le LiDAR sousestime généralement la hauteur réelle (Heurich et al., 2004).

La différence entre la hauteur dominante mesurée par I'IPRFW actualisée à l'année du passage LiDAR (HdommAJ) et la variable Hdom LiDAR a ainsi été calculée pour chaque placette du jeu de calibration. Cette différence est nommée HdomBiais. La possible influence de la pente du terrain, de l'essence, de l'âge des peuplements et de Hdom LidAR $_{\text {sur la valeur de Hdom }}$ Biais a ensuite été analysée à l'aide de la fonction $\mathrm{glm}$ (modèle linéaire généralisé) du logiciel statistique R ( $R$ development Core Team, 2011). Nous avons préféré cette méthode à une modélisation directe de la hauteur dominante de manière à pouvoir identifier précisément les variables influant sur la différence entre la hauteur dominante et la variable Hdom LiDAR $_{\text {étant entendu que }}$ l'existence d'une corrélation entre la hauteur dominante et Hdom LiDAR peut être considérée comme évidente.

Les conclusions de ces analyses ont servi à la mise au point d'un modèle permettant l'obtention d'une estimation non biaisée de la hauteur dominante à partir de Hdom LiDAR. La qualité de ce modèle a été évaluée sur le jeu de données de validation.

\subsection{Forestimator : un plugin QGIS d'estimation de données forestières}

\section{Description générale de l'outil}

Dans le but de diffuser les estimations fournies par les modèles produits, un plugin d'extraction automatique de données a été développé dans le système d'information géographique open source QGIS. Ce plugin se veut convivial et simple afin d'être facilement utilisable par les personnes intéressées à connaître la hauteur dominante de peuplements forestiers (propriétaires, gestionnaires, chercheurs, etc.).

Le plugin réalise de manière automatique une estimation de la hauteur dominante, en appliquant le modèle présenté au paragraphe précédent, à des polygones définis par l'utilisateur. Ces polygones, censés correspondre à des limites de parcelles, doivent être stockés sous format shapefile.

L'estimation de la hauteur dominante fournie par le plugin est accompagnée de la date d'acquisition des données LiDAR et du coefficient de variation des maxima locaux utilisés pour calculer Hdom LiDAR (équation 1)

$$
\mathrm{CV}=\sigma_{\text {HmaxLocaux }} / \text { Hdom }_{\text {LiDAR }}
$$

où $\sigma_{H \max L o c a u x}$ est l'écart-type des hauteurs des maxima locaux intervenant dans le calcul de Hdom LiDAR.

Des valeurs élevées de CV traduiraient une hétérogénéité importante des hauteurs au sein du peuplement. Cette hétérogénéité pourrait résulter d'une délimitation géographique non adéquate, englobant par exemple, deux peuplements distincts.
Un indice de qualité de l'estimation est également produit (Qual). II correspond au rapport entre le nombre

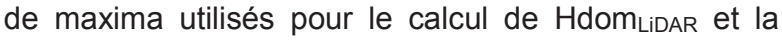
surface, exprimée en ares, du polygone délimitant le peuplement (équation 2) :

$$
\text { Qual }=\operatorname{nmax} /(100 * \mathrm{~S})
$$

où nmax est le nombre de maxima locaux utilisés dans le calcul de Hdom LiDAR et $S$ est la surface du polygone en hectare.

Lorsque le nombre de tiges présentes dans le peuplement est suffisant, ce ratio vaut 1 . Des valeurs inférieures à 1 traduisent des situations où le nombre de tiges est inférieur à 100 par hectare et pour lesquelles la notion de hauteur dominante peut devenir moins pertinente.

Si la table d'attributs du fichier de polygones contient la date de plantation et l'identification de l'essence plantée, le plugin procède à une estimation de la hauteur dominante actualisée à la date de la requête et à une estimation du Site Index. Ces calculs s'appuient sur les modèles de croissance de la hauteur dominante développés par Perin et al. $(2013 ; 2014)$ pour les peuplements équiennes d'épicéa et de douglas (équation 4).

$$
\begin{aligned}
\text { Hdom }= & {\left[\mathrm{a} *\left(\operatorname{age}-\operatorname{age}_{\mathrm{M}}\right)+\left(\frac{\mathrm{Hdom}_{\mathrm{M}}}{\left[1-\exp \left(-\frac{\mathrm{age}_{\mathrm{M}}}{\mathrm{c}}\right)\right]^{\mathrm{r}}}\right)\right] * } \\
& {\left[1-\exp \left(-\frac{\mathrm{age}}{\mathrm{c}}\right)\right]^{\mathrm{r}} \text { (éq 4.) } }
\end{aligned}
$$

où $a, c$ et $r$ sont les paramètres ajustés pour chaque essence dans ces deux études; Hdom-age est le couple hauteur-âge à estimer; Hdom $_{M}$-age $\mathrm{M}_{M}$ est le couple hauteur-âge mesuré ; les âges sont tous estimés depuis la germination.

Dans le cas présent, Hdom $_{M}$ est la hauteur dominante estimée à partir du LiDAR et age $\mathrm{M}_{\mathrm{M}}$, l'âge des arbres constituant le peuplement à la date d'acquisition des données LiDAR. Afin de respecter la gamme de validité des modèles préconisée par leurs auteurs, l'actualisation de la hauteur dominante et le calcul du Site Index ne sont effectués que pour des peuplements de plus de 20 ans.

\section{Interface de l'outil}

L'interface du plugin Forestimator comporte une liste déroulante permettant d'identifier la couche cartographique (fichier shapefile) contenant les polygones à traiter (Figure 2). Une rubrique optionnelle permet de définir la dimension d'une distance tampon (buffer) qui peut être utilisée pour éliminer la partie périphérique du polygone lors de l'estimation de la hauteur dominante. Cette option peut s'avérer utile lorsque la précision géométrique des polygones laisse à désirer et que des effets de bord sont susceptibles d'altérer la qualité de l'estimation.

Deux autres listes déroulantes optionnelles permettent d'identifier au sein de la table d'attributs de la couche de polygones, les champs correspondant à la date de plantation et à l'essence plantée. Ces deux informations 
complémentaires permettent d'actualiser la hauteur dominante au moment de l'exécution de la requête et de calculer un Site Index.

Lorsqu'il s'exécute, le plugin fonctionne selon une architecture classique client-serveur (Figure 3). Pour chaque polygone (parcelle) contenu dans le shapefile, le plugin envoie les données relatives à la géométrie (en format WKT) ainsi que les attributs complémentaires (distance du buffer, date de plantation, essence) au serveur (1).

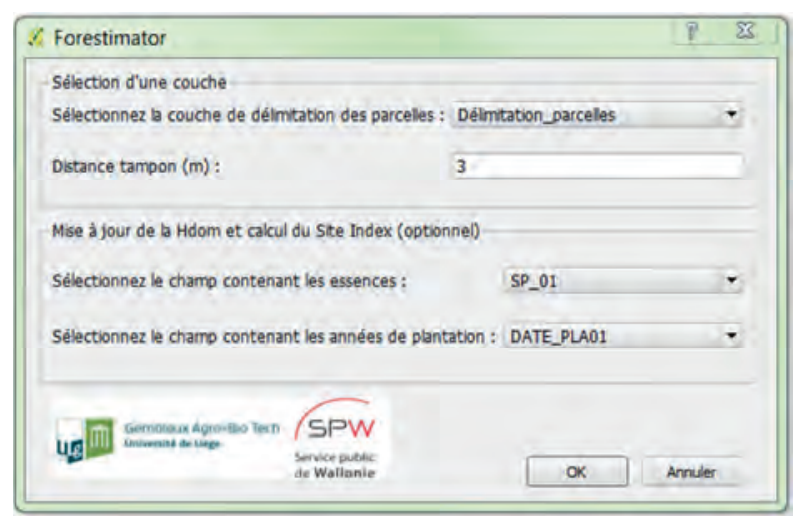

Figure 2 : Interface du plugin Forestimator.

Au niveau du serveur, une requête est exécutée sur une base de données PostGIS qui contient l'information sur l'ensemble des maxima locaux détectés pour la Wallonie. Elle permet d'extraire les 100 plus grands maxima locaux à l'hectare pour chaque polygone et de calculer la hauteur dominante associée $(2 \& 3)$.

Si les données optionnelles (date de plantation et essence) ont été fournies, le serveur calcule également la hauteur dominante actualisée ainsi que le Site Index (4) avant de renvoyer ces résultats vers le client (5). Ces données sont finalement utilisées par le plugin QGIS pour calculer l'intervalle de confiance autour de la hauteur dominante, le coefficient de variation et l'indice de qualité avant d'actualiser la table d'attributs du shapefile pour chaque polygone (6).

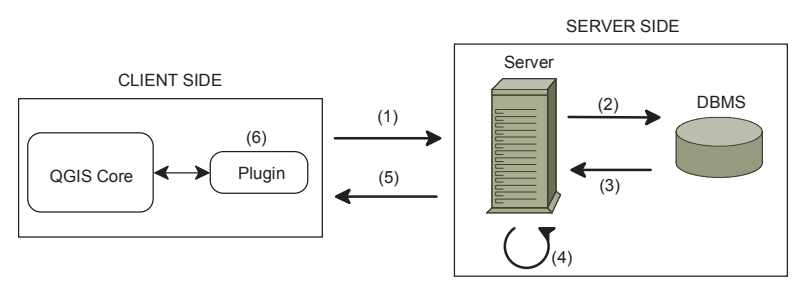

Figure 3 : Schéma de fonctionnement du plugin selon une architecture client-serveur: (1) envoi des limites des polygones; (2\&3) exécution d'une requête pour calculer la hauteur dominante de chaque polygone; (4) si la date de plantation et l'essence sont renseignées, actualisation de la hauteur dominante et calcul du Site Index; (5) renvoi des résultats vers le client; (6) calcul du coefficient de variation et de l'indice de qualité au niveau du plugin QGIS.

\section{Résultats}

\subsection{Estimation de la hauteur dominante}

L'exécution de la fonction CanopyMaxima sur le MNC conduit à une liste reprenant pour chaque maximum local, sa position et sa hauteur associée. Au total, plus de 100 millions de maxima locaux ont été détectés pour la Wallonie.

Le LiDAR sous-estime de façon significative la hauteur

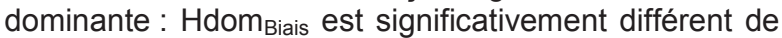
zéro pour les deux essences (Tableau 3). En outre,

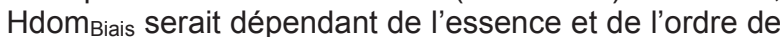
$1,0 \mathrm{~m}$ pour l'épicéa et de $1,3 \mathrm{~m}$ pour le douglas, ces deux valeurs étant significativement différentes l'une de l'autre.

\begin{tabular}{lcc}
\hline & $\begin{array}{c}\text { Epicéa } \\
(\mathrm{n}=225)\end{array}$ & $\begin{array}{c}\text { Douglas } \\
(\mathrm{n}=32)\end{array}$ \\
\hline Hdom $_{\text {MAJ }}$ & $24,80 \pm 0,58$ & $26,95 \pm 1,96$ \\
Hdom $_{\text {LiDAR }}$ & $23,78 \pm 0,58$ & $25,66 \pm 1,92$ \\
Hdom $_{\text {Biais }}$ & $1,02 \pm 0,10$ & $1,29 \pm 0,23$ \\
\hline
\end{tabular}

Tableau 3 : Comparaison, pour chaque essence, des moyennes des hauteurs dominantes calculées dans les placettes de I'IPRFW mises à jour à la date de l'acquisition LiDAR (Hdom MAJ ), des moyennes des hauteurs dominantes estimées par le LiDAR

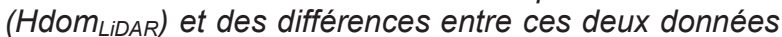
(Hdom Biais . Les valeurs en italique correspondent aux intervalles de confiance (risque d'erreur de type I de 5 $\%)$.

L'application d'une procédure de type best-subset (choix de la meilleure combinaison de variable) n'a permis d'identifier aucune relation statistiquement significative au seuil des $5 \%$ entre la variable Hdom $_{\text {Biais }}$ et Hdom LiDAR, la pente du terrain ou l'âge du peuplement. La table des coefficients correspondant à l'ajustement du modèle linéaire généralisé intégrant toutes les variables (sans interaction avec l'essence) pour modéliser la valeur de Hdom Biais est fournie à titre d'exemple (Tableau 4).

\begin{tabular}{|c|c|c|c|c|}
\hline & Estimate & $\begin{array}{l}\text { Std. } \\
\text { Error }\end{array}$ & $\begin{array}{c}\mathrm{t}- \\
\text { value }\end{array}$ & $\operatorname{Pr}(>|t|)$ \\
\hline (Intercept) & 1,34994 & 0,18700 & 7,219 & $1,92 \mathrm{e}-12$ *** \\
\hline $\begin{array}{l}\text { Essence } \\
\text { (Douglas) }\end{array}$ & 0,38620 & 0,15420 & 2,505 & 0,0126 * \\
\hline Hdom $_{\text {LiDAR }}$ & $-0,02430$ & 0,01278 & $\begin{array}{c}- \\
1,902\end{array}$ & 0,0577 \\
\hline $\begin{array}{l}\text { Pente } \\
\text { terrain }\end{array}$ & $-0,01068$ & 0,00712 & $\begin{array}{c}- \\
1,499\end{array}$ & 0,1345 \\
\hline Age & 0,00721 & 0,00449 & 1,606 & 0,1088 \\
\hline
\end{tabular}

Tableau 4 : Tableau des coefficients de l'ajustement linéaire généralisé intégrant toutes les variables (sans interaction avec l'essence).

Ainsi, seule l'essence aurait un effet significatif sur la

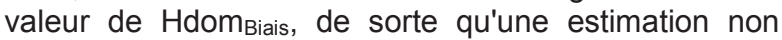
biaisée de la hauteur dominante peut être obtenue à partir de la relation (équation 3):

$$
\text { Hdom }=\text { Hdom }_{\text {LiDAR }}+\text { Hdom }_{\text {Biais }}
$$

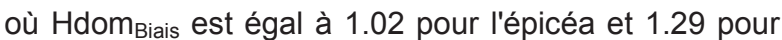
le douglas (Tableau 3).

Les critères statistiques de performance du modèle sont présentés dans le Tableau 5. L'erreur moyenne non significativement différente de 0 indique que les estimations obtenues en appliquant le modèle sur le jeu de données de validation sont non-biaisées. La valeur 
de l'écart-type résiduel est de l'ordre du mètre, ce qui indique que l'intervalle de confiance autour des estimations de la hauteur dominante serait de l'ordre de $\pm 2 \mathrm{~m}$. Le modèle permet d'expliquer respectivement $97,2 \%$ et $98,7 \%$ de la variation de la hauteur dominante dans les peuplements d'épicéa et de douglas.

\begin{tabular}{lcccc}
$\begin{array}{l}\text { Jeu de } \\
\text { données }\end{array}$ & $\mathbf{N}$ & $\begin{array}{c}\text { Écart-type } \\
\text { résiduel }(\mathbf{m})\end{array}$ & $\begin{array}{c}\text { Erreur } \\
\text { moyenne }(\mathbf{m})\end{array}$ & $\mathbf{R}^{\mathbf{2}}$ \\
\hline Epicéa & & & & \\
Calibration & 449 & 1,04 & - & 0,972 \\
Validation & 225 & 1,08 & $0,03 \pm 0,14$ & 0,970 \\
\hline Douglas & & & & \\
Calibration & 65 & 0,93 & - & 0,987 \\
Validation & 32 & 1,33 & $-0,18 \pm 0,46$ & 0,980 \\
\hline
\end{tabular}

Tableau 5 : Valeurs pour chaque essence de l'écarttype résiduel, de l'erreur moyenne et du coefficient de détermination correspondant à l'application du modèle d'estimation de la hauteur dominante à partir des mesures LiDAR sur les jeux de données de calibration et de validation.

\subsection{Le plugin Forestimator : exemple d'utilisation}

A titre d'illustration, le plugin Forestimator a été utilisé pour décrire les parcelles résineuses de la forêt communale de Gedinne située dans le Sud-Ouest de la Wallonie (bloc 3), le long de la frontière française. Cette forêt comprend 1166 ha de plantations d'épicéa, 320 ha de plantations de douglas et 566 ha de mélange de ces deux essences. Ces 2052 ha sont répartis en 404 pessières, 298 douglasaies et 202 peuplements de mélange de ces essences. Les limites de chaque peuplement sont connues et disponibles sous format shapefile. La date de plantation, ainsi que l'identification des essences plantées sont renseignées dans la table d'attributs.

Au total, le plugin s'est exécuté en moins de 2 minutes pour parcourir les 904 peuplements. Un buffer de 3 mètres a été appliqué pour atténuer les erreurs liées à une mauvaise correspondance entre les limites de peuplements et le parcellaire.

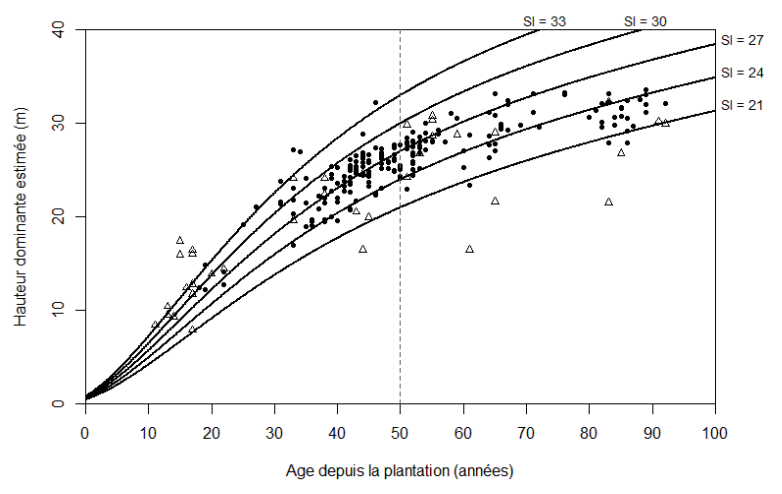

Figure 4: Distribution des hauteurs dominantes estimées en fonction de l'âge depuis la plantation pour les 404 pessières de la forêt communale de Gedinne. Les parcelles présentant un indice de qualité inférieur à 1 ou un coefficient de variation supérieur à $15 \%$ sont représentées par des triangles. Les courbes de productivités (Perin et al., 2014) permettent d'évaluer le potentiel de production des peuplements.

La Figure 4 reprend la distribution des hauteurs dominantes estimées en fonction de l'âge des pessières situées dans cette forêt communale. Les courbes matérialisent les 5 classes de productivité définies pour cette essence en Wallonie par Perin et al. (2014). Elles correspondent à des hauteurs dominantes à 50 ans (Site Index) respectivement de 33, 30, 27, 24 et 21 mètres.

Afin de tester la sensibilité de l'algorithme de calcul de la hauteur dominante à la surface des parcelles, les 50 parcelles les plus étendues (surface moyenne = 8,0 ha ; écart-type $=2,4$ ha) ont été découpées manuellement en 400 sous-parcelles (surface moyenne $=1,0$ ha ; écart-type $=0,16$ ha), afin de se rapprocher de la surface de référence considérée dans la définition théorique. La hauteur dominante a été calculée pour chaque sous-parcelle, puis agrégée à l'échelle des parcelles, en pondérant par la surface des sous-parcelles. Les hauteurs dominantes ainsi obtenues ont été comparées aux valeurs originales, fournies sans découpage des parcelles. Les valeurs originales sont systématiquement supérieures aux valeurs résultant de l'agrégation des hauteurs dominantes locales. La différence moyenne est de $0,30 \mathrm{~m}$ (écart-type $=0,19 \mathrm{~m}$ ), l'écart maximum de 1,0 m étant observé pour la parcelle la plus étendue (16,5 ha).

\section{Discussion}

Le LiDAR est désormais utilisé de manière opérationnelle en appui à la gestion forestière dans différents pays (White et al., 2013a; Naesset, 2007 ; Maltamo et al. 2011; Woods et al. 2011). Aucune utilisation dans ce sens n'existe actuellement en Belgique où ce type de données est apparu récemment. Le plugin Forestimator fournit une information précise et facile d'accès et s'inscrit ainsi dans une démarche de transfert de connaissances entre la recherche et l'opérationnel.

La hauteur dominante est souvent utilisée pour estimer le potentiel de production des peuplements purs équiennes. II est donc important que son estimation soit robuste car elle intervient de manière récurrente dans les outils d'aide à la gestion (par exemple dans les tables de production ou les tables de cubage). Nos résultats indiquent que la technique de détection de maxima locaux est fiable et précise pour estimer la hauteur dominante dans les peuplements réguliers d'épicéa et de douglas, en accord avec les conclusions de Popescu et al. (2002). Ainsi, la précision des estimations de hauteur dominante obtenue (écart-type résiduel d'environ $1 \mathrm{~m}$ ) est tout à fait compatible avec l'utilisation qui est faite de ce type de données. On peut en outre penser que l'amplitude de l'intervalle de confiance autour d'une estimation soit inversement proportionnelle à la taille de la parcelle analysée, de sorte que l'estimation soit en fait plus précise pour des surfaces supérieures à celle des placettes de I'IPRFW (0,1 ha).

La définition de la hauteur dominante que nous avons utilisé (Rondeux, 1999) considère implicitement une surface de référence de 1 hectare. Nous avons pu montrer qu'en présence de parcelles de taille supérieure (de 3 à 17 ha pour les 50 parcelles les plus étendues de la forêt communale de Gedinne), la surestimation reste peu importante : elle est inférieure ou égale à $0,5 \mathrm{~m}$ dans près de $90 \%$ des cas. II convient cependant de rester prudent lors de l'utilisation 
de l'outil pour des parcelles de surface supérieure à 10 ha.

Les données de terrain utilisées pour calibrer le modèle proviennent de placettes d'échantillonnage de taille nettement inférieure à 1 ha (généralement de l'ordre de 0,1 ha). On pourrait donc être tenté de baser le fonctionnement de l'algorithme de ForEstimator sur ces surfaces réduites. Une telle approche nécessiterait de gérer correctement le découpage de parcelles parfois très irrégulières en un ensemble uniforme de sousunités de 0,1 ha et de forme carrée ou hexagonale (Birch et al., 2007). Bien que cette solution ne soit pas exempte de difficultés techniques, elle aurait le mérite d'uniformiser l'échelle de prise en compte de la hauteur dominante, entre les sources de données «terrain » et « télédétection ».

Indépendamment de cette notion d'échelle, il est important d'insister sur le fait que la qualité des estimations de hauteur dominante à l'échelle de l'unité de peuplement, fournies par ce type d'outil, dépend très fortement de la précision et de la pertinence des limites de peuplements. Les imprécisions sur le tracé peuvent en parties être gommées à l'aide de l'option « buffer » de ForEstimator. Les parcelles comportant des peuplements de composition ou de stade de développement différents sont, dans une grande mesure détectés, à l'aide du coefficient de variation des maxima locaux. Néanmoins, les objets cartographiques doivent être les plus précis possible au plan géométrique et homogènes au plan sylvicole afin d'éviter de produire des estimations de hauteur dominantes erronées. Par ailleurs, si l'on considère le cas fréquent des peuplements où l'épicéa et le douglas sont en mélange pied par pied, on peut admettre que les arbres dominants sont généralement des douglas, et que les estimations de hauteur dominante et de Site Index peuvent être réalisées en considérant exclusivement cette essence.

Nous avons observé que l'essence avait une influence significative sur la valeur de la différence entre la hauteur dominante mesurée sur le terrain et la hauteur dominante LiDAR. II est possible, voire probable, que d'autres variables aient également une influence sur cette différence mais qu'elles soient trop faibles ou trop peu variables pour être détectables avec notre jeu de données. Le modèle développé est actuellement limité à l'épicéa et au douglas mais pourrait être facilement adapté à d'autres essences à condition de disposer des jeux de données de calibration et des modèles de croissance en hauteur dominante nécessaires.

Ce nouvel outil, en plus de fournir aisément des estimations de hauteur dominante, propose des indicateurs qui permettent d'évaluer la pertinence de l'estimation d'une hauteur dominante au sein d'un peuplement. L'analyse de la distribution de ces indicateurs dans nos jeux de données montre par exemple que $95 \%$ des placettes sont caractérisées par un coefficient de variation des maxima locaux détéctés sur les données lidar inférieur ou égal à $7,5 \%$ et que l'estimation de la hauteur dominante semble généralement moins précise dans les placettes où sa valeur dépasse $15 \%$. Des valeurs supérieures à cette limite pourraient indiquer des peuplements hétérogènes ou irréguliers. L'indice de qualité, lorsqu'il est inférieur à
1 , indique pour sa part des peuplements où la densité détectée est inférieure à 100 arbres par hectare et où le calcul d'une hauteur dominante peut être contesté.

L'innovation principale de ce plugin réside dans l'intégration de modèles de croissance en hauteur dominante et d'estimation de Site Index avec des estimations de hauteur dominante dérivées de données LiDAR aérien. Cette association permet d'une part d'actualiser l'information de hauteur dominante au cours du temps sans répéter les acquisitions LiDAR qui sont encore relativement coûteuses et d'autre part, d'estimer directement le niveau de productivité d'un peuplement lorsque l'âge et l'essence sont renseignés par l'utilisateur. Un point sensible de l'utilisation de ces modèles de croissance concerne les peuplements mis à blanc entre deux acquisitions LiDAR. Le couplage des données LiDAR avec des jeux de données d'imagerie aérienne (White et al., 2013b) ou satellitaire acquis plus régulièrement permettrait de détecter les mises à blancs, notamment via la construction de $\mathrm{MNH}$ mixte (MNS photogrammétrique - MNT LiDAR). Actuellement, il est supposé que l'utilisateur du plugin dispose d'une information actualisée concernant les limites et l'état des parcelles forestières auxquelles il s'intéresse.

L'estimation du Site index des peuplements équiennes d'épicéa et de douglas permettra d'établir des cartes de productivité de ces essences sur l'entièreté d'un territoire régional ce qui est encore inhabituel à cette échelle (Bock et al. 2009). Cette importante quantité de données pourrait ainsi être employée pour mettre au point des modèles autécologiques robustes et précis permettant ainsi de définir une stratégie optimale pour le choix des peuplements à maintenir ou des essences à planter dans les surfaces dédiées à la forêt. Cette information pourra également intervenir en supplément du fichier écologique des essences qui détermine, pour les principales essence de production en Wallonie, les exigences écologiques, la productivité, ou encore les risques en matière de maladies (Claessens et al., 2014).

A une échelle plus locale, le plugin peut être utilisé en appui à la gestion et aux inventaires forestiers en évaluant facilement le niveau de développement et le potentiel de production des peuplements résineux d'un massif. L'exemple de la forêt communale de Gedinne montre comment le plugin permet, presque instantanément, de classifier les peuplements en fonction de leur niveau de productivité et ainsi aider à la mise au point d'itinéraires de gestion adaptés à la vitesse de croissance de chaque peuplement.

\section{Conclusion et perspectives}

Cet article présente Forestimator, un outil innovant de caractérisation de la ressource forestière. II combine un modèle exploitant des données de type LiDAR aérien à des modèles de croissance en hauteur dominante pour fournir des estimations actualisées de hauteur dominante et de Site Index pour les deux principales essences résineuses de production plantées en Wallonie. L'outil, validé à l'échelle de cette région, fonctionne dans l'environnement du logiciel open source QGIS et peut être apparenté à un Web 
Processing Service. L'environnement SIG permet à l'utilisateur de bénéficier de toutes les fonctionnalités d'un tel logiciel (import/export de données, analyses spatiales, création de cartes, etc), pour valoriser les données fournies par ce service. Gratuit et facile d'utilisation, ce plugin est destiné tant aux gestionnaires forestiers qu'aux scientifiques. Actuellement disponible pour les peuplements d'épicéa et de douglas, son applicabilité sera prochainement étendue à d'autres essences de production.

L'exploitation des produits de base dérivés du vol LiDAR (MNT, MNS) a permis d'obtenir des résultats intéressants pour l'estimation de la hauteur dominante des peuplements résineux. Cependant, les premières analyses réalisées pour l'estimation d'autres paramètres dendrométriques tels que la surface terrière ou encore le volume semble peu prometteuse en utilisant uniquement ces données synthétiques. L'utilisation directe du nuage de points (fichier .las) ou de variables dérivées via une approche raster (Treitz et al., 2012) ou voxel (Reitberger et al., 2009) devraient conduire à des résultats significatifs pour l'estimation de ces paramètres dendrométriques importants pour le gestionnaire forestier.

\section{Remerciements}

Ce travail a été financé par le Service Public de Wallonie - DGRNE à travers le projet «Accord Cadre de Recherche et Vulgarisation Forestières (ACRVF) ». Les auteurs remercient également $M$. Hugues Lecomte, M. Matthieu Alderweireld et M. Mickhail Pitchugin de I'Inventaire Permanent des Ressources Forestières de Wallonie (IPRFW) qui ont fourni les données nécessaires à la calibration et à la validation des modèles.

\section{Références}

Bastin L., Mclnerney D., Revez G., Figueiredo C., Simonetti D., Barredo J., Achard F., San-Miguel-Ayanz, J., 2012. Web Services for Forest Data, Analysis and Monitoring: Developments from EuroGEOSS. IEEE Earthzine, 5(2): 389531.

Birch, C.P., Oom, S.P., Beecham, J.A., 2007. Rectangular and hexagonal grids used for observation, experiment and simulation in ecology. Ecological Modelling 206(3-4): 347-359.

Bock J., Dambrine E., Dez G., Dupouey J.-L., GeorgesLeroy M., Jolly A., Martins F.S.R.V., Renaud, J.-P., 2009. Towards site index mapping in deciduous stands using multi-echo LiDAR data. In IUFRO Division 4 Meeting - Extending Forest Inventory and Monitoring over Space and Time, Quebec, Canada.

Claessens H., Bifolchi E., Bythell S., Cordier S., De Bont A., Desjonquères A., lboukassene $S$., Ridremont F., Van Der Perre R., Vincke C., Ponette Q., 2014. Le nouveau fichier écologique des essences. Pourquoi et comment? Forêt Wallonne, 129 : 60-70.

Coops N. C., Wulder M. A., Culvenor D. S., St-Onge B., 2004. Comparison of forest attributes extracted from fine spatial resolution multispectral and LiDAR data. Canadian Journal of Remote Sensing, 30(6): 855866.

Hägglund B., 1981. Evaluation of forest site productivity. In Forestry Abstracts, 42(11): 515-527.

Heurich M., Persson Å., Holmgren J., Kennel E., 2004. Detecting and measuring individual trees with laser scanning in mixed mountain forest of central Europe using an algorithm developed for Swedish boreal forest conditions. International Archives of Photogrammetry, Remote Sensing and Spatial Information Sciences, 36: 307-312.

Holmgren J., Nilsson M., Olsson H., 2003. Estimation of tree height and stem volume on plots using airborne laser scanning. Forest Science, 49(3): 419-428.

Kaartinen H., Hyyppä J., Yu X., Vastaranta M., Hyyppä H., Kukko A., Holopainen M., Heipke C., Hirschmugl M., Morsdorf F., Næsset E., Pitkäenen J., Popescu S., Solberg S., Wolf B. M., Wu J.-C., 2012. An international comparison of individual tree detection and extraction using airborne laser scanning. Remote Sensing, 4(4): 950-974.

McGaughey R. J., 2013. FUSION/LDV: Software for LiDAR data analysis and visualization. February 2013FUSION Version 3.30. USDA Forest Service. Pacific Northwest Research Station, University of Washington, Seattle, WA, USA.

Maltamo M., Packalén P., Suvanto A., Korhonen K. T., Mehtätalo L., Hyvönen P., 2009. Combining ALS and NFI training data for forest management planning: a case study in Kuortane, Western Finland. European Journal of Forest Research, 128(3): 305-317.

Maltamo M., Packalén P., Kallio E., Kangas J., Uuttera J., Heikkilä J., 2011. Airborne laser scanning based stand level management inventory in Finland. Proceedings of the 11th International Conference on LiDAR Applications for Assessing Forest Ecosystems, University of Tasmania, Australia, 16-20 October 2011. $10 \mathrm{pp}$.

Morgenroth J., Visser R., 2013. Uptake and barriers to the use of geospatial technologies in forest management. New Zealand Journal of Forestry Science, 43(1): 16.

Næsset E., 1997. Estimating timber volume of forest stands using airborne laser scanner data. Remote Sensing of Environment, 61(2): 246-253.

Næsset E., Bjerknes K. O., 2001. Estimating tree heights and number of stems in young forest stands using airborne laser scanner data. Remote Sensing of Environment, 78(3): 328-340.

Næsset E., 2004. Practical large-scale forest stand inventory using a small-footprint airborne scanning laser. Scandinavian Journal of Forest Research, 19(2): 164-179.

Næsset E., 2007. Airborne laser scanning as a method in operational forest inventory: status of accuracy assessments accomplished in Scandinavia. Scandinavian Journal of Forest Research, 22(5): 433442. 
Næsset E., 2014. Area-Based Inventory in Norway-From Innovation to an Operational Reality. In Forestry Applications of Airborne Laser Scanning, 215-240.

Nilsson M., 1996. Estimation of tree heights and stand volume using an airborne lidar system. Remote Sensing of Environment, 56(1): 1-7.

Office Économique Wallon du Bois, 2013. PanoraBois Wallonie 2012-2013. Editeur Emmanuel Defays, Marche-en-Famenne.

Perin J., Hébert J., Brostaux Y., Lejeune P., Claessens $\mathrm{H}$., 2013. Modelling the top-height growth and site index of Norway spruce in Southern Belgium. Forest Ecology and Management, 298: 62-70.

Perin J., De Thier O., Claessens H., Lejeune P., Hébert J., 2014. Nouvelles courbes de productivité harmonisées pour le douglas, l'épicéa et les mélèzes en Wallonie. Forêt Wallonne, 129 : 26-41.

Persson A., Holmgren J., Söderman U., 2002. Detecting and measuring individual trees using an airborne laser scanner. Photogrammetric Engineering and Remote Sensing, 68(9): 925-932.

Popescu S. C., Wynne R. H., Nelson R. F., 2002. Estimating plot-level tree heights with lidar: local filtering with a canopy-height based variable window size. Computers and Electronics in Agriculture, 37(1): 71-95.

Popescu S. C.,Wynne R. H., 2004. Seeing the trees in the forest: using lidar and multispectral aata fusion with local filtering and variable window size for estimating tree height. Photogrammetric Engineering and Remote Sensing, 70(5): 589-604.

Reitberger J., Schnörr C., Krzystek P., Stilla U., 2009. 3D segmentation of single trees exploiting full waveform LIDAR data. ISPRS Journal of Photogrammetry and Remote Sensing, 64(6): 561-574.

Rondeux J., 1999. La mesure des arbres et des peuplements forestiers. Les presses agronomiques de Gembloux, Gembloux.
Rondeux J., Lecomte H., 2010. Inventaire Permanent des Ressources Forestières de Wallonie: Guide méthodologique. SPW, DG03, Jambes.

$\mathrm{R}$ development Core Team, 2011. R: a language and environment for statistical computing. R Foundation for Statistical Computing, Vienna, Austria. http://www.rproject.org. 27 March 2015.

Harma M., Amateis R.L., Burkhart H.E., 2002. Top height definition and its effect on site index determination in thinned and unthinned loblolly pine plantations. Forest Ecology and Management, 168: 163-175.

Treitz P., Lim K., Woods M., Pitt D., Nesbitt D., Etheridge D., 2012. LiDAR sampling density for forest resource inventories in Ontario, Canada. Remote Sensing, 4(4): 830-848.

Watt P., Meredith A., Yang C., Watt M. S., 2013. Development of regional models of Pinus radiata height from GIS spatial data supported with supplementary satellite imagery. New Zealand Journal of Forestry Science, 43(1): 11.

White J. C., Wulder M. A., Varhola A., Vastaranta M., Coops N. C., Cook B. D., Pitt D., Woods M. 2013a. A best practices guide for generating forest inventory attributes from airborne laser scanning data using an area-based approach. Natural Resources Canada, Canadian Forest Service, Canadian Wood Fibre Center, Victoria, BC. Information Report FI-X-010 https://cfs.nrcan.gc.ca/publications?id=34887

White J. C., Wulder M. A., Vastaranta M., Coops N. C. Pitt D., Woods M., 2013b. The utility of image-based point clouds for forest inventory: A comparison with airborne laser scanning. Forests, 4(3): 518-536.

Woods M., Pitt D., Penner M., Lim K., Nesbitt D., Etheridge D., Treitz P., 2011. Operational implementation of a LiDAR inventory in Boreal Ontario. The Forestry Chronicle, 87(4): 512-528. 